\title{
THE CHARACTERISTIC OF A QUADRATIC FORM FOR AN ARBITRARY FIELD
}

\author{
BY \\ RUFUS OLDENBURGER
}

1. Introduction. Ernst Witt [1] $\left.{ }^{(}\right)$has shown that for a field $K$ with characteristic not 2 each quadratic form $Q$ can be transformed into a form $G+H$ where

$$
G=\sum_{i=1}^{\sigma}\left(x_{i}^{2}-y_{i}^{2}\right),
$$

$H$ is a nonzero form, that is, does not represent zero properly, the rank of $Q$ is the sum of the ranks of $G$ and $H$, and $G$ has rank $2 \sigma$. The number $\sigma$ is an invariant of $Q$ under nonsingular linear transformations on $Q$. For the real field the number $\sigma$ was defined by Loewy [2] as the minimum of the indices $\left({ }^{2}\right)$ of $Q$ and $-Q$, and termed the characteristic of $Q$. Loewy showed that this characteristic could be defined in terms of exponents of elementary divisors of pencils $\{\rho F-Q\}$ formed from $Q$ and real quadratic forms $\{F\}$. The definition of Loewy does not extend to an arbitrary field $K$, whereas the characteristic of $Q$ for $K$ is arrived at by Witt through the examination of a sequence of quadratic forms $Q, H_{1}, \cdots, H_{\sigma}$, where for each $s$ the form $Q$ is $G_{s}+H_{s}$ for

$$
G_{\varepsilon}=\sum_{i=1}^{s}\left(L_{i}^{2}-M_{i}^{2}\right),
$$

the $L$ 's and $M$ 's being linearly independent linear forms, while the rank of $Q$ is the sum of the ranks of $G_{s}$ and $H_{s}$. In the present paper we shall show that the characteristic of $Q$ can be defined in terms of linearly independent linear forms directly associated with $Q$. This definition is particularly convenient for treating sums of forms.

With the aid of the viewpoint developed here it is proved ( $\$ \$ 3-4)$ that the characteristic of a quadratic form $Q$ is related to the ranks of the principal minors of matrices associated with $Q$. By means of this relation we are able to treat the characteristics of sums of forms, and to show in particular (\$5) that the characteristic of $Q+\lambda L^{2}, L$ linear, differs at most by 1 from that of $Q$. This property is likewise possessed by the rank of $Q$, and, if $K$ is real, also by the index of $Q$. The above result on the characteristic of $Q+\lambda L^{2}$ will be used in another paper [3] to show that the characteristic $\sigma$ of a quadratic

Presented to the Society, April 3, 1942; received by the editors February 13 and September 2, 1942.

(1) The numbers in brackets refer to the bibliography at the end of the paper.

(2) The index of $Q$ is the number $h$ of + signs in a canonical form $x_{1}^{2}+\cdots+x_{h}^{2}-x_{h+1}^{2}$ $\ldots-x_{r}^{2}$ to which $Q$ is equivalent under a nonsingular linear transformation. 
form $Q$ determines the minimum value $\tau$ for which $Q$ can be written as

$$
\sum_{i=1}^{\tau} L_{i} M_{i}
$$

where the $L$ 's and $M$ 's are linear forms. The maximum value which can be attained by the characteristic of $Q$ relative to the rank $r$ of $Q$ is $[r / 2]$, where $[r / 2]$ designates the largest integer not exceeding $r / 2$.

A sum

$$
Q=\sum_{i=1}^{r} \lambda_{i} L_{i}^{2}
$$

where the $L$ 's are linear, and $r$ is the rank of $Q$, is a minimal representation of $Q$.

2. Preliminary definitions and conventions. Throughout the present paper the usual restriction that the characteristic of the field $K$ be different from 2 is made in order that each quadratic form $Q$ may be written as

$$
Q=\sum_{i, j=1}^{n} a_{i j} x_{i} x_{j}
$$

where the matrix $\left(a_{i j}\right)$ of coefficients is symmetric. We term $\left(a_{i j}\right)$ the matrix $A$ of $Q$.

In what follows we shall use the term "equivalent" to mean equivalent under nonsingular linear transformations.

We define the characteristic of a quadratic form $Q$ to be the maximum number $\sigma$ of linearly independent linear forms $L_{1}, \cdots, L_{\sigma}$ such that the rank of

$$
Q+\lambda_{1} L_{1}^{2}+\cdots+\lambda_{\sigma} L_{\sigma}^{2}
$$

is identical with the rank of $Q$ for all values of the $\lambda$ 's. In what follows the term "characteristic" will be understood to refer to the invariant just defined, until we have proved $(\$ 3)$ that this invariant is identical with the characteristic of Witt, and for the real field with that of Loewy.

If a form $Q$ with rank $r$ is written as a sum $G+H$ where $G$ has rank $2 \sigma$ and characteristic $\sigma$, and $H$ has rank $r-2 \sigma$ and characteristic 0 , we term $G+H$ a characteristic splitting of $Q$. If we write $Q$ as

$$
\sum_{i=1}^{\sigma} L_{i} M_{i}+H
$$

where the component in the $L$ 's and $M$ 's is identical with the form $G$ in a characteristic splitting of $Q$, we have a decomposition of $Q$.

If the rank of the form (2.2) is identical with the rank $r$ of $Q$ for all values of the $\lambda$ 's, the forms $L_{1}, \cdots, L_{\sigma}$ clearly depend only on the variables which occur in $Q$. A stronger statement can be made. We let 


$$
\mu_{1} M_{1}^{2}+\cdots+\mu_{r} M_{r}^{2}
$$

be a minimal representation of $Q$. Since $M_{1}, \cdots, M_{r}$ may be taken as the variables in terms of which $Q$ is expressed, it follows that the $L$ 's are linear forms in the $M$ 's, whence the characteristic of $Q$ cannot exceed $r$.

3. Characteristic splittings. In the present section we shall relate the characteristic of a quadratic form $Q$ to the results of Witt.

We recall that a matrix $D$ of order $c$ and rank $d$ has nullity $c-d$.

LEMMA 3.1. The characteristic of a quadratic form $Q$ of rank $r$ is the maximum $\sigma$ for which $Q$ is equivalent to a form $F$ with rth order matrix $C$ where complementary principal minors $C_{11}, C_{22}$ of $C$ have order and nullity $\sigma$, respectively.

In considering matrices $\{C\}$ with complementary principal minors $C_{11}$ and $C_{22}$, it will be no restriction if we take $C_{11}$ to be a leading minor so that

$$
C=\left\|\begin{array}{ll}
C_{11} & C_{12} \\
C_{21} & C_{22}
\end{array}\right\| \text {. }
$$

We write $Q$ as in (2.1) with $n=r$. We suppose that $Q$ has characteristic $\sigma$. We may assume without restriction that the rank of (2.2) with $L_{i} \equiv x_{i}$ for each $i$ is identically equal to $r$ for all values of the $\lambda$ 's. We assume that $\sigma \geqq 1$. We let $M$ denote the minor of the matrix $A$ of $Q$ obtained from $A$ by deleting the first $\sigma$ rows and $\sigma$ columns of $A$. Expanding the determinant of the form (2.2) we find that the rank of the form (2.2) is $r$ for all values of the $\lambda$ 's if and only if each principal minor determinant of $A$ containing $M$ vanishes, except the determinant $|A|$ itself. The rank of $M$ is a number $b$, where $b<r-\sigma$. We may suppose that $M$ is in the shape

$$
\left\|\begin{array}{ll}
D & 0 \\
0 & 0
\end{array}\right\|
$$

where $D$ is a nonsingular minor of order $b$. We suppose, for the moment, that $b \geqq r-2 \sigma$, and consider the minor determinants of $A$ of the type

$$
\left|\begin{array}{lll}
* & * & E^{\prime} \\
* & D & 0 \\
E & 0 & 0
\end{array}\right|,
$$

where $E$ has order $r-\sigma-b$, and $E^{\prime}$ denotes the transpose of $E$. If $b>r-2 \sigma$, the minors of type (3.3) are distinct from $|A|$. Since the vanishing (3.3) implies the singularity of $E$, the last $r-\sigma-b$ columns of $A$ are linearly dependent, a contradiction. Thus $b \leqq r-2 \sigma$. If $b<r-2 \sigma$, the matrix $A$ is singular. Thus $b=r-2 \sigma$.

Conversely, if $Q$ has the matrix $C$ where $C_{22}$ has rank $r-2 \sigma$ and order $r-\sigma$, we adjoin elements from $q$ rows and $q$ columns of $C$ to $C_{22}$ to obtain a minor 
of $C$ of order $m$, where $m=r-\sigma+q$, with rank at most $r^{\prime}$, where $r^{\prime}=r-2 \sigma+2 q$. If $\sigma>0$, the relation $q<\sigma$ implies that $r^{\prime}<m$, whence each square minor of $C$ containing $C_{22}$, except $C$, is singular. The rank of (2.2) with $L_{i} \equiv x_{i}$ for each $i$ is now $r$ for all choices of the $\lambda$ 's, whence the characteristic of $Q$ is at least $\sigma$.

THEOREM 3.1. A quadratic form $Q$ with rank $r$ has characteristic $\sigma$ if and only if $Q$ has the characteristic splitting $G+H$, where $G$ has rank $2 \sigma$ and characteristic $\sigma$, while $H$ has rank $r-2 \sigma$ and characteristic 0 .

By a result of Witt, quoted in the introduction, the form $Q$ is equivalent to a sum

$$
\sum_{i=1}^{p} u_{i} v_{i}+H
$$

where $H$ is a nonzero form with rank $r-2 \rho$. The number $\rho$ (by the theory of Witt) is uniquely determined by $Q$. Further, if $Q$ is equivalent to a form (3.4) where $H$ is a zero form with rank $r-2 \rho$, the form $Q$ is equivalent to a sum (3.4) with $\rho$ replaced by a larger number $\rho^{\prime}$, where $H$ now is a nonzero form with rank $r-2 \rho^{\prime}$.

We let $\sigma$ denote the characteristic of $Q$. Since the rank of

$$
Q+\lambda_{1} u_{1}^{2}+\cdots+\lambda_{\rho} u_{\rho}^{2}
$$

is $r$ for all values of the $\lambda$ 's we have $\sigma \geqq \rho$.

By Lemma 3.1 the form $Q$ is equivalent to a form $Q^{\prime}$ with the matrix $C$, given in (3.1), where the order of $C_{11}$ and the nullity of $C_{22}$ equal $\sigma$. In view of the nullity of $C_{22}$ we may assume that $C_{22}$ is written as (3.2) where the order of $D$ is equal to $r-2 \sigma$. We write $Q^{\prime}$ as $(2.1)$ with $n=r$, whence $\left(a_{i j}\right)=C$. The form $Q^{\prime}$ is now the sum $G^{\prime}+H^{\prime}$, where

$$
G^{\prime}=\sum_{i=1}^{\sigma} x_{i} L_{i},
$$

the $L$ 's are linear forms and $H^{\prime}$ is the form with matrix $D$. Since $Q^{\prime}$ has rank $r$, the variables $x_{1}, \cdots, x_{\sigma}$ in $G^{\prime}$, as well as $x_{\sigma+1}, \cdots, x_{r-\sigma}$ in $H^{\prime}$, and $L_{1}, \cdots, L_{\sigma}$ comprise a set of linearly independent forms, whence these may be taken as the variables in terms of which $Q^{\prime}$ is expressed. It follows from the Witt theory that $\rho \geqq \sigma$, whence $\rho=\sigma$.

It is readily seen that the component in the $x$ 's and $L$ 's in (3.5) has characteristic $\sigma$, whereas $H^{\prime}$ has characteristic 0 .

From Theorem 3.1 there are a number of immediate consequences valid for an arbitrary field $K$. The characteristic of a quadratic form $Q$ of rank $r$ does not exceed $[r / 2]$. The characteristic of a quadratic form $Q$ of rank $r$ attains the maximum value $r / 2$ if and only if $Q$ is equivalent to the canonical form 


$$
\sum_{i=1}^{r / 2} x_{i} y_{i}
$$

A quadratic form $Q$ is a nonzero form if and only if the characteristic of $Q$ is 0 .

For the complex field the characteristic of a quadratic form $Q$ is $[r / 2]$. For the real field the characteristic is clearly the minimum of the indices of $Q$ and $-Q$. The index and characteristic of $Q$ may thus be distinct. In fact the concept of index of $Q$ is identical with that of characteristic and the type $( \pm)$ of definiteness of a nonzero component $H$ in a canonical splitting of $Q$.

From a result of Dickson [4] one can readily show that the quadratic forms

$$
Q=\sum_{i=1}^{n} \alpha_{i} x_{i}^{2}, \quad E=\sum_{i=1}^{n} e_{i} y_{i}^{2},
$$

with $\alpha_{1}=e_{1}$ and rank $n$, are equivalent if and only if the subforms

$$
\sum_{i=2}^{n} \alpha_{i} x_{i}^{2}, \quad \sum_{i=2}^{n} e_{i} y_{i}^{2}
$$

are equivalent. Witt [1] proved this same result by different methods, and showed that quadratic forms with canonical splittings $G+H$ and $G^{\prime}+H^{\prime}$, where $H$ and $H^{\prime}$ are nonzero forms, are equivalent if and only if they have the same characteristic, and $H$ is equivalent to $H^{\prime}$. Thus $H$ is uniquely determined up to equivalence. The study of the equivalence of quadratic forms thus reduces to that of nonzero forms, so extensively treated in the literature $\left({ }^{3}\right)$.

4. Characteristics and principal minors. To treat characteristics of sums of forms we shall need some properties of principal minors developed here.

LEMma 4.1. If the matrix of order $r$ of a quadratic form $Q$ of rank $r$ has a principal minor of nullity $\sigma$, the characteristic of $Q$ is at least $\sigma$.

Without restriction on the generality of the method we may suppose that the matrix of order $r$ of $Q\left(x_{1}, \cdots, x_{r}\right)$ is given by

$$
A=\left\|\begin{array}{lll}
D_{11} & D_{12} & D_{13} \\
D_{21} & D_{22} & 0 \\
D_{31} & 0 & 0
\end{array}\right\|,
$$

where the bottom right zero represents a minor of order $\sigma$, and $D_{22}$ is nonsingular. We let $t$ designate the number of rows of $D_{13}$. By Lemma 3.1 we may restrict ourselves to the case where $t>\sigma$. By a nonsingular linear transformation affecting only the variables $x_{1}, \cdots, x_{t}$, the form $Q$ can be brought into a form $Q^{\prime}$ with matrix $B$, where the principal minor of $B$ obtained by

(3) See, for example, [5]. 
striking out the first $t$ rows and columns of $B$ is identical with this minor for $A$, and $D_{13}$ is replaced by a nonsingular $\sigma$ th order minor followed by rows of zeros. The matrix $B$ is of the type $C$ given in (3.1), where $C_{11}, C_{22}$ have order and nullity $\sigma$, respectively, whence by Lemma 3.1 the characteristic of $Q$ is at least $\sigma$.

In Lemma 3.1 we showed how the characteristic $\sigma$ of a quadratic form $Q$ is the maximum value $\sigma$ for which certain minors $C_{11}, C_{22}$ possess given properties. We shall show how a further examination of these minors reveals whether or not the maximum value $\sigma$ is attained for them. In the following theorem the characteristic of $C_{22}$ is understood to be the characteristic of the quadratic form associated with $C_{22}$.

THEOREM 4.1. Suppose that the order and nullity of complementary principal minors $C_{11}, C_{22}$ of the matrix of order $r$ of a quadratic form $Q$ of rank $r$ equal $\sigma$, respectively. The characteristic of $Q$ is $\sigma$ if and only if the characteristic of $C_{22}$ is 0 .

We write $Q$ as in $(2,1)$ with $n=r$. Since $C_{22}$ has nullity $\sigma$ there is a nonsingular matrix $M$ such that $M C_{22} M^{\prime}$ is the minor (3.2) where $D$ has order $r-2 \sigma$. It will thus be no restriction on the generality of the method to suppose that the matrix $A$ of $Q$ has the shape (4.1) with $D_{22}=D$, and the order of $D_{11}$ equal to $\sigma$. We can thus write $Q$ as a sum $G^{\prime}+H^{\prime}$, where $G^{\prime}$ is given by (3.5), $H^{\prime}$ is a form with the matrix $D$, and the rank of $Q$ is the sum of the ranks of $G^{\prime}$ and $H^{\prime}$. The characteristic of $G^{\prime}$ is clearly $\sigma$.

If $Q$ has characteristic $\sigma$, the sum $G^{\prime}+H^{\prime}$ is a characteristic splitting of $Q$, whence $H^{\prime}$ has characteristic 0 . It follows that $C_{22}$ has characteristic 0 .

If conversely, the characteristic of $C_{22}$ is 0 , the sum $G^{\prime}+H^{\prime}$ is again a canonical splitting, whence the characteristic of $Q$ is $\sigma$.

We consider the canonical splitting $G+H$, where

$$
G=\sum_{i=1}^{\sigma} x_{i} x_{r-\sigma+i}
$$

and $H$ is a form in $x_{\sigma+1}, \cdots, x_{r-\sigma}$. Since the matrix of $G+H$ is of the type (3.1) with the order of $C_{11}$ and the nullity of $C_{22}$ equal to $\sigma$, and the characteristic of $C_{22}$ equal to 0 , the characteristic of a quadratic form $Q$ is $\sigma$ if and only if $Q$ is equivalent to a form with the matrix (3.1) where complementary principal minors $C_{11}$ and $C_{22}$ have the properties just mentioned.

5. The characteristic of a sum of forms. Each quadratic form $Q$ is equivalent to a quadratic form with a diagonal matrix. This is the same as the property that each quadratic form $Q$ has a minimal representation. It follows that the study of the effect of the addition of a quadratic form $F$ to a quadratic form $Q$ reduces to the study of the addition of a term $\lambda L^{2}, L$ linear, to $Q$.

THEOREM 5.1. Under addition of a term $\lambda L^{2}$, L linear, to a quadratic form $Q$ the characteristic $\sigma$ of $Q$ changes at most by 1 . 
We suppose that the characteristic of $Q+\lambda L^{2}$ is at least $\sigma+2$. We let $q$ designate the rank of $Q+\lambda L^{2}$. By Lemma 3.1 the pair $(Q, L)$ can be transformed nonsingularly into a pair $\left(Q^{\prime}, M\right)$, where the matrix $C$ of $Q^{\prime}+\lambda M^{2}$ is of order $q$, and is of the shape (3.1), while $C_{11}$ and $C_{22}$ have order and nullity $\sigma+2$, respectively.

The rank of a quadratic form $Q$ changes at most by 1 under addition of a term $\lambda L^{2}, L$ linear, to $Q$. We shall assume, to begin with, that $q=r$, where $r$ is the rank of $Q$. The addition of $-\lambda M^{2}$ to $Q^{\prime}+\lambda M^{2}$ changes the nullity of $C_{22}$ by at most 1 . Thus $C_{22}$ goes into a minor $D_{22}$ with nullity at least $\sigma+1$. By Lemma 4.1 the characteristic of $Q^{\prime}$ is at least $\sigma+1$, a contradiction. It follows that the characteristic $t$ of $Q+\lambda L^{2}$ does not exceed $\sigma+1$. Thus

$$
\sigma+1 \geqq t \geqq \sigma-1 \text {. }
$$

We now consider the case where $q=r+1$, and designate the variables in $Q^{\prime}+\lambda M^{2}$ by $y_{1}, \cdots, y_{r+1}$ where the rank of

$$
Q^{\prime}+\lambda M^{2}+\lambda_{1} y_{1}^{2}+\cdots+\lambda_{\sigma+2} y_{\sigma+2}^{2}
$$

is $r+1$ for all values of $\lambda_{1}, \cdots, \lambda_{\sigma+2}$. By the development used in the proof of Lemma 3.1 the form $Q^{\prime}+\lambda M^{2}$ has a matrix $C$ of order $r+1$ as given in (3.1) where $C_{11}$ and $C_{22}$ are minors of order and nullity $\sigma+2$, respectively. If $M$ is linearly independent of $y_{1}, \cdots, y_{\sigma+2}$, we may suppose that $M \equiv y_{\sigma+3}$. Removal of the row and column of $C$ corresponding to $y_{\sigma+3}$ yields the matrix of $Q^{\prime}$ of order $r$. The minor obtained from $C_{22}$ by removal of this row and column has nullity at least $\sigma+1$. By Lemma 4.1 the characteristic of $Q^{\prime}$ is at least $\sigma+1$, a contradiction. If, on the other hand, the form $M$ is linearly dependent on $y_{1}, \cdots, y_{\sigma+2}$, the minor $C_{22}$ of $C$ is a minor of a matrix $C^{*}$ of order $r+1$ of $Q^{\prime}$. Since $C_{22}$ has nullity $\sigma+2$, there is a nonsingular matrix $N$ such that $N^{\prime} C^{*} N=A$, where $A$ is given in (4.1) the minors $D_{13}$ and $D_{31}$ being of order $\sigma+2$, while $D_{22}$ is nonsingular. Since $A$ is singular, $D_{13}$ is singular. It follows that there is a nonsingular matrix $M$ such that $M^{\prime} A M$ is identical in shape with $A$ except that the last column of $D_{13}$ is replaced by a column of zeros, and a corresponding remark holds for the last row of $D_{31}$. We drop the last row and column of $M^{\prime} A M$ to obtain a matrix $B$ whose lower principal minor of order $r-\sigma-3$ has nullity $\sigma+1$. By Lemma 4.1 and the invariance of the characteristic under nonsingular linear transformations we have again arrived at a contradiction. Thus in any event, when $q=r+1$, the characteristic of $Q+\lambda L^{2}$ does not exceed $\sigma+1$.

Since the index of $Q$ is $\sigma$ there are linearly independent linear forms $L_{1}, \cdots, L_{\sigma}$ such that the rank of (2.2) is $r$ for all values of the $\lambda$ 's. If $q=r+1$, the form $L$ is linearly independent of the variables in $Q$, whence the rank of

$$
Q+\lambda L^{2}+\lambda_{1} L_{1}^{2}+\cdots+\lambda_{\sigma} L_{\sigma}^{2}
$$


is $r+1$ for all values of $\lambda_{1}, \cdots, \lambda_{\sigma}$. Thus if the rank of $Q+\lambda L^{2}$ exceeds the rank of $Q$ the characteristic of $Q+\lambda L^{2}$ is at least as great as that of $Q$.

The case where $q=r-1$ reverts to the preceding.

COROLlaRY 5.1. If $\rho$ and $R$ denote the characteristic and rank of the quadratic forms $Q$ and $F$, respectively, the characteristic of $Q+F$ satisfies the inequalities:

$$
\rho-R \leqq \sigma \leqq \rho+R .
$$

We shall show that Theorem 5.1 is valid if we replace the characteristic of $Q$ by the index of $Q$ for the real field. In the following theorem all coefficients are understood to be in the real field.

TheOREM 5.2 (Analogue of Theorem 5.1). Under addition of a term $\lambda L^{2}$, $L$ linear, to a real quadratic form $Q$, the index of $Q$ changes at most by 1 .

It is readily seen that Theorem 5.2 is true when the ranks of $Q$ and $Q+\lambda L^{2}$ are distinct. We suppose, therefore, that these ranks are identical. We have

$$
Q+\lambda L^{2} \equiv M_{1}^{2}+\cdots+M_{q}^{2}-M_{q+1}^{2}-\cdots-M_{r}^{2}
$$

where $r$ is the rank of $Q$, while $q$ is the index of $Q+\lambda L^{2}$, and the $M$ 's are linear forms. We suppose that $q>h+1$, where $h$ is the index of $Q$. We may suppose that $Q$ is written as

$$
\sum_{i=1}^{h} x_{i}^{2}-\sum_{i=h+1}^{r} x_{i}^{2}
$$

We set $x_{1}=\cdots=x_{h}=0$. Since $M_{1}, \cdots, M_{q}$ are linearly independent to begin with, at most $h$ of these vanish. We may assume, therefore, that $M_{h+1}$ and $M_{h+2}$ are linearly independent of $x_{1}, \cdots, x_{h}$. We also set $L=M_{q+1}=\cdots$ $=M_{r}=0$. We have

$$
-\sum_{i=h+1}^{r} x_{i}^{2} \equiv \sum_{i=h+1}^{q} M_{i}^{2}
$$

We have imposed at most $r-q+h+1$ independent conditions on the variables in $Q$. It follows that the left and right members in (5.1) do not vanish identically, whence we have a contradiction.

The theorems above and well known rank theory now imply that the rank, characteristic, and, if the field $K$ is real, also the index of a quadratic form $Q$, have the common property that they change at most by 1 under addition of a term $\lambda L^{2}, L$ linear, to $Q$. 


\section{BibliogRaPHY}

1. Ernst Witt, Theorie der quadratischen Formen in beliebigen Körpern, J. Reine Angew. Math. vol. 176 (1937) pp. 31-44.

2. Alfred Loewy, Ueber Scharen reeller quadratischer und Hermitescher Formen, ibid. vol. 122 (1900) pp. 53-72.

3. Rufus Oldenburger, Expansions of quadratic forms, Bull. Amer. Math. Soc. vol. 49 (1942) pp. 136-141. 115.

4. Leonard E. Dickson, On quadratic forms in a general field, ibid. vol. 14 (1907) pp. 108-

5. Helmut Hasse, Über die Darstellbarkeit von Zahlen durch quadratischen Formen im Körper der rationalen Zahlen, J. Reine Angew. Math. vol. 152 (1923) pp. 129-148.

ILLINOIS INSTITUTE OF TECHNOLOGY, Chicago, Ill. 